\title{
Fabrication and Structural Studies of Porous Anodic Oxide Film on Pure Aluminium and Aluminium Alloy (AA 1100)
}

\author{
NAVEEN VERMA ${ }^{1 *}$, KRISHAN CHANDER SINGH ${ }^{1}$, \\ BERNABE MARI $^{2}$ and JITENDER ${ }^{1}$ \\ ${ }^{1}$ Department of Chemistry, Maharshi Dayanand University, Rohtak-124 001, India \\ ${ }^{2}$ Departament de Física Aplicada, \\ Universitat Politècnica de València, 46022 València, Spain \\ vermanaveen17@gmail.com
}

Received 17 September 2013 / Accepted 6 October 2013

\begin{abstract}
Pure aluminium and aluminium alloy 1100 samples were anodized by one step and two step process. The foils were anodised in $0.3 \mathrm{M}$ sulphuric acid solution at constant voltage of $25 \mathrm{~V}$ and the temperature was maintained at $20{ }^{\circ} \mathrm{C}$. The effect of increase of exposure time in second anodization step on the structural features was studied on AA 1100. A well ordered nanoporous structure were produced. It was found that in two step anodization process the produced film have very regular hexagonal cells with uniform pores as compare to single step anodization in both $\mathrm{Al}$ and AA 1100. The two step anodization improves both pore diameter and uniformity. With the increase in second step anodization time the complexities of porous film increases due to formation of sub pores.
\end{abstract}

Keywords: Anodization, Aluminium, AA 1100, Sulphuric Acid

\section{Introduction}

In the past decade researchers have focussed particular attention on the fabrication of porous anodic alumina. Nanochannels array of alumina are of increasing interest in view of their potential application in energy, magnetic, electronic devices and sensing devices ${ }^{1-4}$. Fabrication of ordered nanoporous alumina facilitates the practical application in nanodevices. Therefore a study of formation process, structural and morphological properties has considerable grown. The PAA membranes become one of the most important templates for the preparations of nanowires ${ }^{5}$, nanotubes ${ }^{6}$ and nanoparticles ${ }^{7}$. These films are also used in filters $^{8}$, optical ${ }^{9}$ and photonic applications ${ }^{10}$. The single step anodization of high purity aluminium at constant voltage has less control on pore diameter, pore spacing and geometry ${ }^{11}$. The two step oxidation technology is best to prepare ordered pore arrays. Highly ordered, well structured hexagonal arrays were produced by two step anodization process ${ }^{12-13}$. It was believed that the repulsive forces at metal/oxide interface promote the formation of well ordered hexagonal pores ${ }^{14}$. The anodizing conditions such as type of electrolyte, anodizing 
potential, current density, temperature and duration of exposure controls the pore diameter, interpore distance, porosity, pore density and other characteristic of the anodic oxide film. Now it is possible to obtain various pore diameters ranging from few nanometers to several hundred nanometers. The pores may be widened by immersing the film in $\mathrm{H}_{3} \mathrm{PO}_{4}$ solution. $\mathrm{H}_{2} \mathrm{SO}_{4}$ is widely used in anodization process and highly ordered porous membranes were produced. The best regime voltage is $25 \mathrm{~V}$ for anodization in sulphuric acid ${ }^{15}$.

Template assisted synthesis is a less expensive and simple way to produce nanomaterials. There is no need of high temperature and high vacuum is required in chemical and physical vapour deposition technique. Porous anodic alumina film possess array of nanopores with remarkable properties such as high pore density, circular pores with controllable pore diameter ${ }^{16}$.

In this paper our aim is to compare the porous anodic film formed on aluminium alloy 1100 with the pure aluminium (99.997). It has great importance because Aluminium alloy has high strength and cheaper than pure aluminium. The effect of anodization time on the pore geometry and pore parameter has been studied. PAA film with highly pore order in both pure aluminium and aluminium alloy 1100 were prepared in $\mathrm{H}_{2} \mathrm{SO}_{4}$ and morphology studies were carried out using Field emission scanning electron microscopy.

\section{Experimental}

A high purity aluminium foil (99.997\%, Alfa Aesar) and commercially available aluminium alloy 1100 (99.5\% purity) were used as starting material. The aluminium 1100 has $98.88 \%$ aluminium with $0.020 \% \mathrm{Cu}, 0.10 \% \mathrm{Zn}, 0.05 \% \mathrm{Mn} \& 0.95 \%$ is $\mathrm{Si}+\mathrm{Fe}$ as alloying element. First of all aluminium substrate were mechanically polished with fine grade emery paper followed chemical etching with $\mathrm{NaOH}(100 \mathrm{~g} / \mathrm{L})$. Then the samples were electro polished in a mixture of $\mathrm{HClO}_{4}: \mathrm{C}_{2} \mathrm{H}_{5} \mathrm{OH}$ (1:5) for 3 minute at constant voltage of $15 \mathrm{~V}$. After electro polishing the samples were successively rinsed with distilled water ethanol and dried. The samples were anodized by one step and two step procedure for the fabrication of alumina film. In the first step the sample were anodised for two hour in $0.3 \mathrm{M} \mathrm{H}_{2} \mathrm{SO}_{4}$ at $25 \mathrm{~V}$. The prepared alumina layer was washed with an etching mixture of $6 \mathrm{wt} \% \mathrm{H}_{3} \mathrm{PO}_{4}$ and $1.8 \mathrm{wt} \%$ $\mathrm{H}_{2} \mathrm{Cr}_{2} \mathrm{O}_{4}$ at $60{ }^{\circ} \mathrm{C}$ for 3 hours. Then the second anodization was performed for $3 \mathrm{~h}, 4 \mathrm{~h}$ and $5 \mathrm{~h}$. The other anodization conditions were same as in first step. Anodization was performed in a simple electrochemical cell with magnetic stirrer under the constant temperature of $20{ }^{\circ} \mathrm{C}$. The working surface of sample was $1 \mathrm{~cm}^{2}$. A Platinum mesh was used as cathode. The samples were placed in centre of the platinum mesh. The aim of this work was to investigate the influence of alloying element on the structural feature and regularity of pore arrangement in $\mathrm{H}_{2} \mathrm{SO}_{4}$ media in AA 1100 .

\section{Results and Discussion}

The current-time characteristics plot has been recorded and shown in Figure 1 for the first step anodization in $0.3 \mathrm{M} \mathrm{H}_{2} \mathrm{SO}_{4}$ at $25 \mathrm{~V}$ for pure aluminium and AA 1100 . In all samples the current time curves shows a local minima within 10 seconds and the minima was followed by a distinct maxima. The minima indicate the growth of compact layer which have high resistance.

The formation of maxima after minima is due to the transformation of compact oxide film in to porous film and then the current density again decreases due to increase in thicknesses which in turn increase the resistance. The maxima in the curve ascribed to the 
formation and dissolution of film and occurring of pore rearrangement process on the surface. The current densities observed during anodization of pure Al and AA1100 differ at the maxima and AA1100 shows the higher current density. This indicates the rapid dissolution of AA1100 as compare to growth of oxide film. The slight retardation in growth process is due to presence of alloying elements in $\mathrm{Al} 1100$. When $\mathrm{Fe}$ and $\mathrm{Mn}$ are the alloying element they affect the film formation characteristics like field assisted film dissolution and conductivity during the formation of oxide film ${ }^{17-18}$.

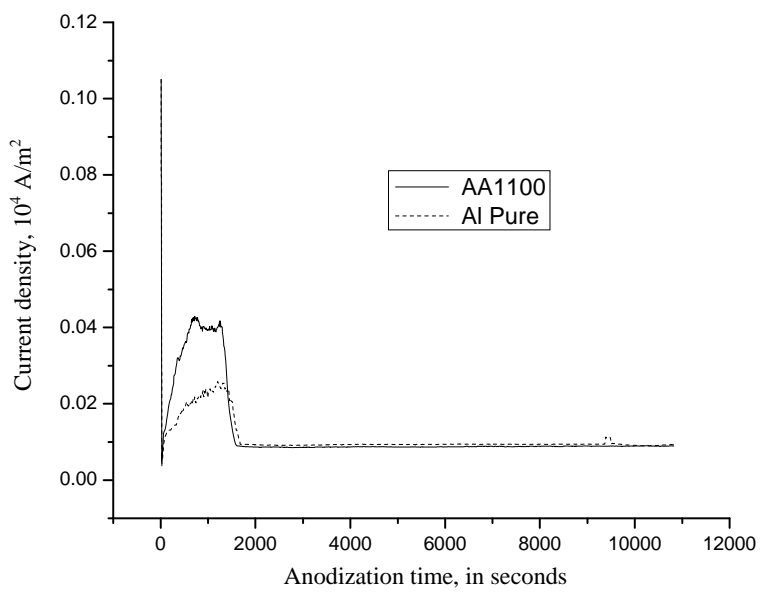

Figure 1. The current time characteristic for anodization of pure and $\mathrm{Al} 1100$

From the literature it has been observed that when Si and Fe species were present as impurity, they hinder the growth of oxide film while anodizing aluminium alloy ${ }^{19}$. The accumulation of alloying element on metal oxide interface influence the anodic oxidation and consequently modifies the oxide microstructure. A regular but dissymmetric porous arrangement were observed for samples of both pure $\mathrm{Al}$ and $\mathrm{AA} 1100$ anodised in $\mathrm{H}_{2} \mathrm{SO}_{4}$ in single step for two hours (Figure 2). But pore density is larger in case of AA 1100. The pores in the film on AA 1100 are broader at the surface and narrow at the bottom having different geometries with diameter ranges from 8 to $25 \mathrm{~nm}$. The anodic oxide film formed on pure $\mathrm{Al}$ has spherical shape pores with pore diameter from 18 to $48 \mathrm{~nm}$. FESEM images in Figure 3 shows the morphological structure of film formed by two step anodization. The porous alumina produced by two step anodization produces a very regular self organised hexagonal array of pores. It is clearly visible that with increase in anodization time the pore diameter increase from 27 to $40 \mathrm{~nm}$ but the interpore distance is almost same.
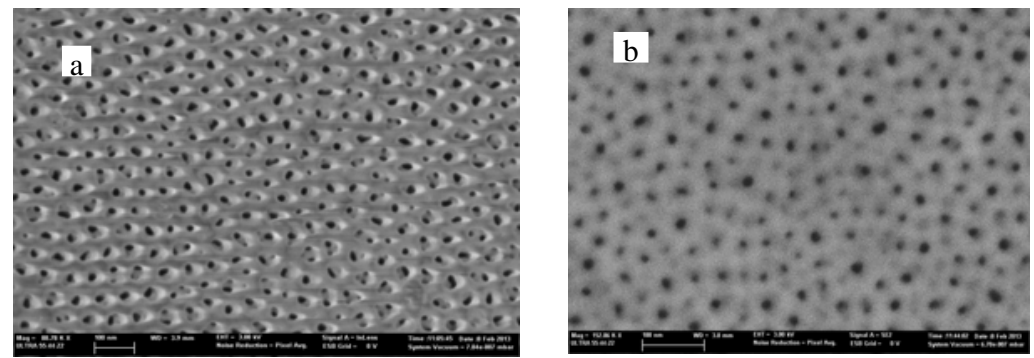

Figure 2. FE-SEM image of anodized in single step in $0.3 \mathrm{M} \mathrm{H}_{2} \mathrm{SO}_{4} \mathrm{Al} 1100$ (A) and high purity aluminium (B) 

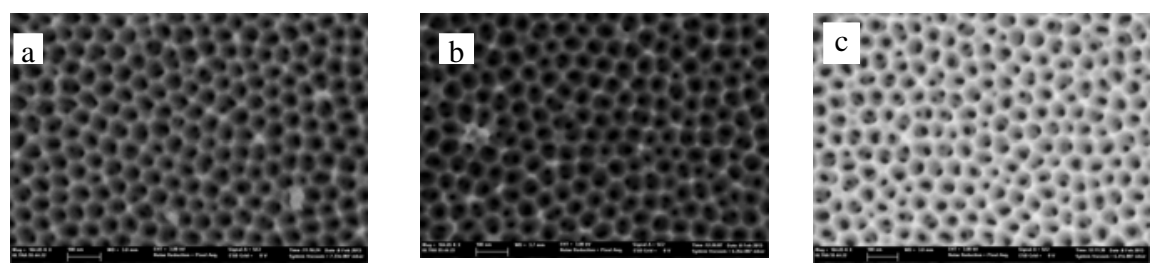

Figure 3. FE-SEM image of $\mathrm{Al} 1100$ anodized in double step in $0.3 \mathrm{M} \mathrm{H}_{2} \mathrm{SO}_{4}$ for; (a) 3 hours, (b) 4 hours, (c) and 5 hours.

This porous anodic alumina film exhibit sub porous structure below the surface. When the Al 1100 samples were anodized in two step anodization processes, the samples were anodized first for two hours then washed in phosphoric acid and chromic acid mixture and then anodized again for 3 hours in the same condition as in first anodization. The two step anodization process on AA 1100 produce very well organised hexagonal honey comb like structure. When second step anodization time increases to $4 \mathrm{~h}$ and $5 \mathrm{~h}$ then the film exhibit a more complex structures with sub pores beneath the surface. The pure aluminium sample anodized in two step process produces the slightly more regular arrangement as compare to AA 1100 samples anodized at the same conditions. With increase in the anodization time in the second step the hexagonally arranged cells clearly visible in the FE-SEM image and produces a more complex structure with increase sub pores concentration. It is clearly visible from the FE-SEM image (Figure 3a. and 4) samples of pure $\mathrm{Al}$ and AA 1100 that there is no large effect of there alloying element in AA 1100 and that can be used in the place of pure aluminium which is very costly as compared to AA 1100.

Figure 5 shows the measured reflectance spectra of AAO films on AA1100 and pure aluminium by one step anodization and AA 1100 double anodized to different extant of time ( $3 \mathrm{~h}, 4 \mathrm{~h}$ and $5 \mathrm{~h}$ ). As a result of anodization, the reflectance of anodised specimens decreased as anodization time increases. It is due to the fact that it is due to the increase in thickness of anodic alumina. Shie et al., ${ }^{20}$ has found that interference start at about $100 \mathrm{~nm}$ of AAO film thickness. The origin of reflectance and colour production for anodised sample may originate from the interference of light waves between the interfaces of light waves between the interfaces of metal AAO membrane and successive AAO films.

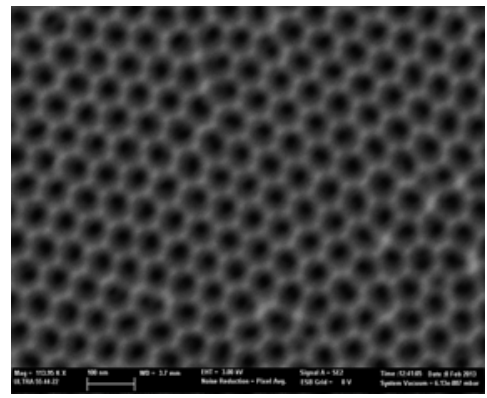

Figure 4. FE-SEM image of pure $\mathrm{Al}$ anodized in double step in $0.3 \mathrm{M}$ $\mathrm{H}_{2} \mathrm{SO}_{4}$ for $3 \mathrm{~h}$

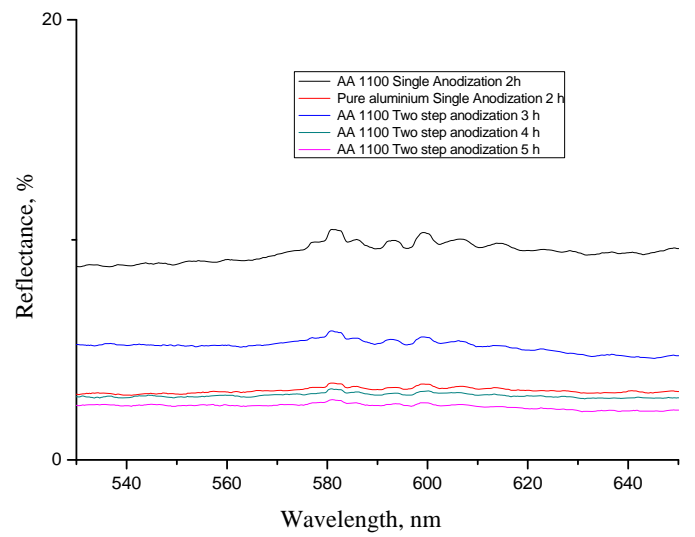

Figure 5. Experimental total reflectance of anodized $\mathrm{Al}$ for different samples 
Thickness of the sample increases with increase in anodization time which in turn increases the capability to absorb the light waves. When the anodic film increases its thickness beyond a critical value, the interference disappears and light wave are likely to be absorbed by the AAO film decreases the total reflectance. The thickness of the samples were calculated and presented in the Table 1

Table 1. Thickness calculated from reflectance measurements

\begin{tabular}{lc}
\hline \multicolumn{1}{c}{ Sample } & Thickness, $\mu \mathrm{m}$ \\
\hline AA 1100 single step anodization 2 $\mathrm{h}$ & 12.80 \\
Al Pure single step anodization 2 $\mathrm{h}$ & 13.54 \\
Al 1100 single two step anodization 3 h & 12.52 \\
Al 1100 single two step anodization 4 h & 12.80 \\
Al 1100 single step anodization 5 h & 13.03 \\
\hline
\end{tabular}

\section{Conclusion}

a) Single step anodization of aluminium 1100 has higher porosity than pure aluminium.

b) A two step self organised anodization of aluminium performed in $0.3 \mathrm{M} \mathrm{H}_{2} \mathrm{SO}_{4}$ at $25 \mathrm{~V}$ results in a complex pore structure with sub pores forms under the film surface and the complexity of sub pores increases with increase in second step anodization time.

c) The $\mathrm{Al} 1100$ is slight less ordered porous structure than pure aluminium but the pore diameter is almost same so used as template for synthesize in nano materials which reduces the cost because $\mathrm{Al} 1100$ is cheaper than highly pure aluminium.

\section{Acknowledgement}

Naveen Verma thanks the University grant commission, Delhi, India for providing financial assistance under UGC major research project 40-77/2011(SR) and Jitender thanks CSIR, New Delhi, India for the award of Junior Research Fellowship.

\section{References}

1. $\quad$ Evans P R, Yi G and Schwarzacher W, Appl Phys Lett., 2000, 76(4), 481-483.

2. Lee J, Kim J and Hyeon T, Adv Mater., 2006, 18(16), 2073-2094;

DOI:10.1002/adma.200501576

3. Vrublevsky I, Jagminas A, Schreckenbach J and Goedel A W, Appl Surface Sci., 2007, 253(10), 4680-4687.

4. Kim Y, Jung B, Lee H, Kim H, Lee K and Park H, Sensors Actuators B: Chem., 2009, 141(2), 441-446; DOI:10.1016/j.snb.2009.07.007

5. Sarkar J, Khan G G and Basumallick A, Bull Mater Sci., 2007, 30(3), 271-290; DOI:10.1007/s12034-007-0047-0

6. Shao X F, Wu X L, Huang G S, Qiu T, Jiang M and Hong J M, Appl Phys A, 2005, 81(3), 621-625; DOI:10.1007/s00339-004-2639-5

7. $\quad$ Lei Y, Cai W and Wilde G, Prog Mater Sci., 2007, 52(4), 465-539; http://dx.doi.org/10.1016/j.pmatsci.2006.07.002

8. $\quad$ Fernando J A and Chung D D L, J Porous Mater., 2002, 9(3), 211-219; ; DOI:10.1023/A:1020991002430

9. Huang C H, Lin H Y, Tzeng Y, Fan C H, Liu C Y, Li C Y, Huang C W, Chen N K and Chui H C, Sensors Actuators A: Phys., 2012,180, 49-54; http://dx.doi.org/10.1016/j.sna.2012.04.001

10. Xu Q, Sun H Y, Yang Y H, Liu L H and Li Z Y, Appl Surf Sci., 2011, 258(5), 18261830; http://dx.doi.org/10.1016/j.apsusc.2011.10.054 
11. Nielsch K, Choi J , Schwirn K, Wehrspohn R B and Gosele U, Nano lett., 2002, 2, 677-680; DOI:10.1021/nl025537k

12. Masuda H and Fukuda K, Science, 1995, 268(5216), 1466- 1468;

DOI:10.1126/science.268.5216.1466

13. Masuda H and Satoh M, Jpn J Appl Phys., 1996, 35, L126-L129;

DOI:10.1143/JJAP.35.L126

14. Jessensky O, Müller F and Gosele U, Appl Phys Lett., 1998, 72(10), 1173-1175.

15. Sulka G D, Nanostructured Materials in Electrochemistry, Wiley-VCH, Weinheim, Chapter, 2008.

16. Zaraska L, Sulka G D, Szerameta J and Jaskula M, Electrochim Acta, 2010, 55(14), 4377- 4386; http://dx.doi.org/10.1016/j.electacta.2009.12.054

17. Fratila L E, Duszczyk J and Katger L, Surf Coat Technol., 2002, 157(1), 80-94; http://dx.doi.org/10.1016/S0257-8972(02)00144-5

18. Mukhopadhyay K and Sharma A K, Surface Coat Technol., 1997, 92(3), 212-220; http://dx.doi.org/10.1016/S0257-8972(97)00102-3

19. Fratila L E A, Ticherlaar F D, Thompson G E, Terryn H, Skeldon P, Duszczyk J and Katerman L, Electrochim Acta, 2004, 49(19), 3169-3177; http://dx.doi.org/10.1016/j.electacta.2004.02.030

20. Teng S S, Wei P S and Huang Y S J, Surf Coat Technol., 2008, 202(14), 3298-3305; http://dx.doi.org/10.1016/j.surfcoat.2007.12.002. 